\title{
Preferencias de docentes universitarios sobre herramientas para plataformas de e-learning
}

\author{
University professors preferences on tools for e-learning \\ platforms
}

\author{
Víctor René García Peña ${ }^{1}$ \\ sercomgar@hotmail.com \\ ORCI: 0000-0002-3088-3559 \\ Carlos Vinicio López Rodríguez 2 \\ litos plus@yahoo.es \\ ORCID: 0000-0003-3434-3768 \\ Alex Bladimir Mora Marcillo 3 \\ bladimormar@gmail.com \\ ORCID: 0000-0003-0202-5642 \\ Henrry Marcelino Pinargote Pinargote 4 \\ henrrypinargote@gmail.com \\ ORCID: 0000-0001-8871-3651 \\ Estefanía Vanessa García Solórzano 5 \\ stefaniavanessa11@gmail.com \\ ORCID: 0000-0003-0110-2183
}

Recibido: 07/12/2020, Aceptado: 26/03/2021

\section{RESUMEN}

Esta investigación tuvo como objetivo determinar las preferencias de docentes universitarios sobre herramientas para plataformas de e-learning. Fue cuantitativa, con diseño no experimental y nivel descriptivo. La población caso de estudio fue de 20 docentes de la Universidad Laica "Eloy Alfaro" de Manabí, Ecuador, que estuvieran utilizando plataformas virtuales para e-learning. Se recolectaron los datos con un cuestionario de seis dimensiones y 45 proposiciones cerradas con cinco alternativas de respuesta y una pregunta abierta. Fue validado y determinada su confiabilidad mediante el alfa de Cronbach, obteniendo 0.932. Como resultados entre las herramientas orientadas al aprendizaje, la preferencia fue el uso de comunicación síncrona (chat); en las orientadas a la productividad, el uso de tutoriales, manuales de usuario, ayuda en línea; para las de implicación de estudiantes, el uso de autenticación de usuarios, mediante nombre de usuario y contraseña; para las destinadas a publicación de cursos y contenidos, el uso del seguimiento del estudiante; para el diseño de planes de estudio, el uso de reutilización y compartición de contenidos y para las de gestión del conocimiento, el uso de sistemas integrales de conocimiento. Se concluyó que los motivos de la no utilización, o uso con poca frecuencia de las herramientas pueden ser diversos.

\footnotetext{
${ }^{1}$ Universidad Laica Eloy Alfaro de Manabí, Ecuador.

${ }^{2}$ Universidad Laica Eloy Alfaro de Manabí, Ecuador.

${ }^{3}$ Universidad Laica Eloy Alfaro de Manabí, Ecuador.

${ }^{4}$ Universidad Laica "Eloy Alfaro" de Manabí, Ecuador.

${ }^{5}$ Universidad San Gregorio de Portoviejo, Ecuador.
} 


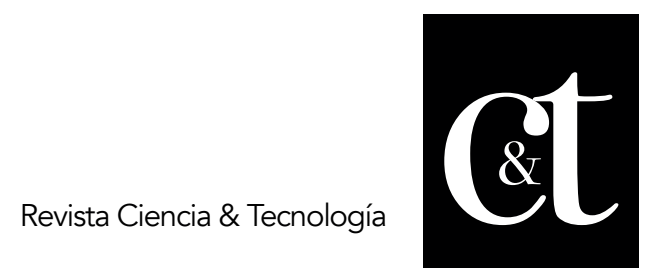

No. 30, 30 de abril de 2021

ISSN impreso: 1390 - 6321

ISSN online: 2661 - 6734

Se recomienda continuar indagando sobre estos aspectos mencionados, así como también las preferencias de los estudiantes.

Palabras clave: Plataformas de e-learning, herramientas para plataformas virtuales, e-learning; docentes universitarios, entornos virtuales.

\begin{abstract}
This research aimed to determine the preferences of university teachers about tools for e-learning platforms. It was quantitative, with a non-experimental design and a descriptive level. The case study population was 20 teachers from the Laica University "Eloy Alfaro" of Manabí, Ecuador, who were using virtual platforms for e-learning. Data were collected with a seven-dimensional questionnaire and 45 closed propositions with five response alternatives and an open question. Its reliability was validated and determined using Cronbach's alpha, obtaining 0.932. As results among the learning-oriented tools, the preference was the use of synchronous communication (chat); in those oriented to productivity, the use of tutorials, user manuals, online help; for student involvement, the use of user authentication, by username and password; for those destined to the publication of courses and contents, the use of student monitoring; for the design of study plans, the use of reuse and content sharing and for knowledge management, the use of comprehensive knowledge systems. It was concluded that the reasons for the non-use or infrequent use of the tools can be diverse. It is recommended to continue inquiring about these aspects mentioned, as well as the preferences of the students
\end{abstract}

Keywords: E-learning platforms, tools for virtual platforms, e-learning; university teachers, virtual environments.

\title{
Introducción
}

Es evidente y comprobado que las tecnologías de la información y comunicación (TIC) han ocupado un lugar importante y trascendental en la vida de toda persona a nivel mundial, hasta el punto de que se han hecho imprescindibles en casi todas las actividades cotidianas, tales como por ejemplo transacciones económicas, interacciones sociales, distracción y por supuesto, en educación, donde han sido utilizadas como herramientas de impacto positivo para la enseñanza y el aprendizaje.

En este sentido, una buena forma de realizar aportes a la calidad del proceso de enseñanza y aprendizaje consiste en adaptar los instrumentos de las TIC y transformarlos en recursos novedosos para estudiantes y docentes, especialmente a nivel universitario, donde según Arroyo et al. (2018) se ha venido desarticulado paulatinamente el paradigma clásico de la educación tradicional, por otro donde el uso de las plataformas educativas ha impulsado el intercambio de conocimientos, y el espacio físico se ha acondicionado para una interacción virtual, tomando como opciones efectivas para potenciar el aprendizaje en los estudiantes el reconocimiento de las funcionalidades y herramientas de plataformas elearning.

\section{1}

García, López, Mora, Pinargote, García. Preferencias de docentes universitarios sobre herramientas para plataformas de e-learning 


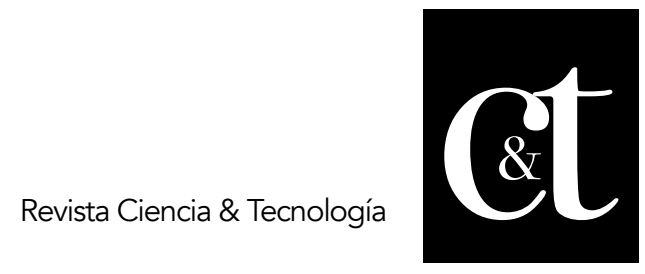

No. 30,30 de abril de 2021

ISSN impreso: 1390 - 6321

ISSN online: 2661 - 6734

La llegada de la Internet cambió la concepción original de educación a distancia, creando un nuevo término: e-learning, o educación en línea, dando paso a los espacios educativos denominados entornos virtuales; siendo necesario adoptar otras formas de comunicación y gestión de recursos de almacenamiento. Generalmente, las actividades de e-Learning se desarrollan en entornos virtuales o plataformas virtuales, denominadas también Learning Management Systems (LMS). Al respecto se tiene que:

El e-learning nació en los años noventa como una modalidad de formación asociada a la educación a distancia y al uso de Internet en la educación superior y la formación empresarial. Conviene destacar que existe una diferencia importante entre los modelos tradicionales de educación a distancia y el elearning. La educación a distancia puede o no utilizar tecnología, pero lo más importante es garantizar el estudio independiente sin necesidad de que haya una intervención continua del docente. En el caso del e-learning, se comparte la no presencialidad del modelo, pero el énfasis se produce en la utilización de Internet como sistema de acceso a los contenidos y a las actividades de la formación. Sirven las plataformas abiertas de Coogle. Además, la interacción y la comunicación son una parte fundamental en este tipo de formación. (Gros, 2018, p.70). En este sentido, la evolución del e-learning ha estado directamente vinculada a las funcionalidades de la Internet, de tal forma que cada día se incorporan cada vez más herramientas para poder diseñar y gestionar cursos individualizados. (Boneu, 2007) estableció cuatro características básicas para cualquier plataforma de e-learning, estas son: interactividad, flexibilidad, escalabilidad y estandarización; además de otras características generales tales como: de código abierto, plataforma gratuita, arquitectura multiidioma, la tecnología empleada y la comunidad de usuarios y documentación.

Entre las herramientas elementales se encuentran las siguientes: a) Herramientas orientadas al aprendizaje; b) orientadas a la productividad; c) para la implicación de los estudiantes; d) de soporte; e) destinadas a la publicación de cursos y contenidos; f) para el diseño de planes de estudio; g) para la gestión del conocimiento en el ámbito educativo (Boneu, 2007). A continuación se describen cada una de ellas.

Se ha usado la Guía de Trabajo de Alejandro Gallardo (2020). Entre las herramientas rumbo al aprendizaje están: los foros de discusión, concebidos como herramientas para intercambiar vía on-line información, preguntas y respuestas, archivos y todo tipo de material sobre diversos temas, también como espacio para discusiones académicas durante el tiempo que dure un curso. Los foros pueden tener buscadores para facilitar la localización de determinados mensajes, entre todos los temas de debate incluyendo el patrón de búsqueda indicado.

Otra herramienta de este tipo es el e-portafolio o portafolio digital, el cual es útil para hacer el seguimiento del aprendizaje, a través del acceso a los trabajos realizados los cuales pueden estar en diferentes formatos. También está el intercambio de archivos, usado para proveer acceso a información almacenada digitalmente, puede ser implementado con distintos tipos de almacenamiento, transmisión y modelos de distribución. Las Herramientas de comunicación síncrona y asíncrona tales como chat, correo electrónico o mensajería para permitir el intercambio comunicativo entre los participantes. 


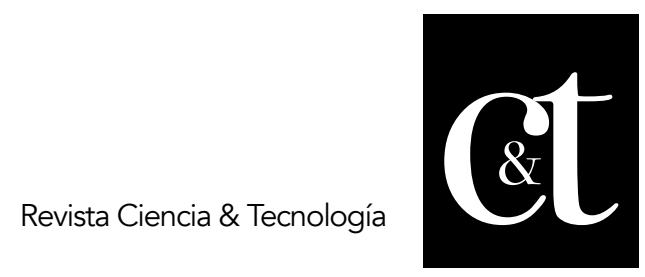

No. 30, 30 de abril de 2021

ISSN impreso: 1390 - 6321

ISSN online: 2661 - 6734

Los servicios de presentación multimedia, tales como videoconferencias, vídeos, pizarras electrónicas, entre otros. Los Diario (blogs) o notas en línea, aplicables en su forma educativa a través de los edublogs. Los Wikis, o herramientas que facilitan la elaboración de documentos en línea de forma colaborativa.

Las herramientas orientadas a la productividad están conformadas por los bookmarks, quienes permiten volver fácilmente a una página web visitada; el calendario y revisión del progreso, útil para la planificación de las actividades de un curso; la ayuda en el uso de la plataforma, tales como tutoriales, manuales de usuario, ayuda en línea; el buscador de cursos mediante un patrón de búsqueda; los mecanismos de sincronización y trabajo fuera de línea, donde los estudiantes tienen la posibilidad de trabajar desconectados de la plataforma; el control de publicación, páginas caducadas y enlaces rotos; las noticias del lugar; los visos de actualización de páginas, mensajes a foros y envío automático, útiles para avisar cada vez que sucede un evento en la plataforma que concierne al usuario; el soporte a la sindicación de contenidos o herramientas para incorporar a la plataforma contenidos de forma sindicada, que son ofrecidos desde el exterior o desde la propia plataforma.

Entre las herramientas para la implicación de los estudiantes se destacan los grupos de trabajo; las autovaloraciones; el rincón del estudiante, o espacios para hacer grupos de estudio, clubes o equipos de trabajo colaborativos; el perfil del estudiante, o espacios para mostrar el trabajo en un curso, anunciarse, mostrar su fotografía, preferencias, temas de interés o información personal. Como herramientas de soporte se encuentran la autenticación de usuarios, mediante el nombre de usuario y contraseña; la asignación de privilegios en función del rol del usuario; el registro de estudiantes; la auditoría, o herramientas que permiten consultar todas las acciones realizadas por los participantes de la plataforma, así como obtener estadísticas sobre su utilización.

Las herramientas destinadas a la publicación de cursos y contenidos están conformadas por los test y resultados automatizados, los cuales permiten al docente crear, administrar y evaluar; la administración del curso, útil para que el profesores tenga control de la progresión de una clase a través del material del curso, también permite a los estudiantes comprobar sus progresos; el apoyo al creador de cursos; las herramientas de calificación en línea; y el seguimiento del estudiante.

Entre las herramientas para el diseño de planes de estudio están la conformidad con la accesibilidad; la reutilización y compartición de contenidos; las plantillas de curso, utilidades para crear la estructura de un curso en línea; la administración del currículum; la personalización del entorno, útil para cambiar la apariencia gráfica de los cursos; las herramientas para el diseño de la educación; la conformidad con el diseño de la educación. 


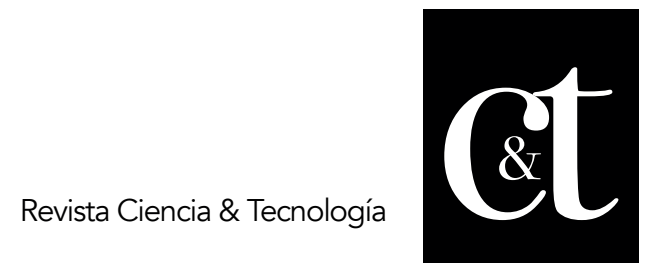

No. 30, 30 de abril de 2021

ISSN impreso: 1390 - 6321

ISSN online: 2661 - 6734

Los Sistemas para la gestión del conocimiento en el ámbito educativo pueden clasificarse en: sistemas integrales de conocimiento; sistemas mediadores de información; librerías digitales o repositorios; los sistemas basados en ontologías; y los sistemas basados en folcsonomías, estos intentan dar un giro importante a la forma en que se clasifica la información. Estas herramientas de LMS han sido utilizadas por docentes universitarios en Ecuador, quienes han desarrollado experiencias de programas educativos con modalidades presenciales, semipresenciales, a distancia o en línea con apoyo de Internet desde el inicio del siglo XXI, experimentando cambios que han sido influenciados por el desenvolvimiento de la sociedad y su vinculación con el desarrollo de las TIC. Así lo manifiestan investigaciones tales como las de Rea y Medina (2016); Guevara, Pardo, e Izquierdo (2017); Martínez et al. (2017); Chávez y Verdezoto (2018); Arroyo et al. (2018), entre otros. Estos cambios son legalmente apoyados por el Consejo de Educación Superior (2019) en el artículo 73 del Reglamento del Régimen Académico del Ecuador quien autoriza la modalidad en línea, expresando:

La modalidad en línea es aquella en la que los componentes de aprendizaje en contacto con el profesor; práctico-experimental; aprendizaje autónomo de la totalidad de los créditos están mediados en su totalidad por el uso de tecnologías interactivas multimedia y entornos virtuales de aprendizaje que organizan la interacción de los actores del proceso educativo, de forma sincrónica o asincrónica, a través de plataformas digitales. (Consejo de Educación Superior, 2019, p.28)

Ahora bien, tal como se describió previamente, es conocido que la mayoría de los entornos virtuales, tanto los de libre acceso como los que no lo son, poseen una amplia variedad de herramientas conducentes al desarrollo de actividades educativas motivantes y novedosas bajo las distintas modalidades, donde el educador se convierte en facilitador, para que el estudiante avance a su ritmo y con sus propios intereses y necesidades.

Estas herramientas son utilizadas por los docentes de distintas formas, según Ilgaz y Gulbahar (2017) y Del Prete y Cabero (2019), la preferencia por el e-learning está relacionada con elementos como la tecnología y el tipo de acción didáctica aplicada, además de algunas características tales como la accesibilidad y flexibilidad que aporta lo virtual en los procesos educativos. Así por ejemplo, en muchos países la plataforma Moodle es la favorita, tal es el caso de Ecuador según lo manifiestan Arroyo et al. (2018). Con lo expuesto hasta ahora se planteó la investigación conducente a la elaboración de este artículo, el cual tiene como objetivo determinar las preferencias de docentes universitarios sobre herramientas para plataformas de e-learning.

\section{Metodología}

Fue realizada una investigación cuantitativa, con diseño no experimental, la cual según Hernández; Fernández y Baptista (2014) se realiza sin manipular las variables, es decir, lo que se hace es observar fenómenos tal como se dan en su contexto natural. Tipo de campo con nivel descriptivo, el propósito de este nivel consiste en la "descripción, registro, análisis e interpretación de la naturaleza actual, composición o procesos de los fenómenos, haciendo énfasis sobre cómo una persona o grupo realizan determinada acción en el presente" (Palella y Martins, 2012, p.92). 


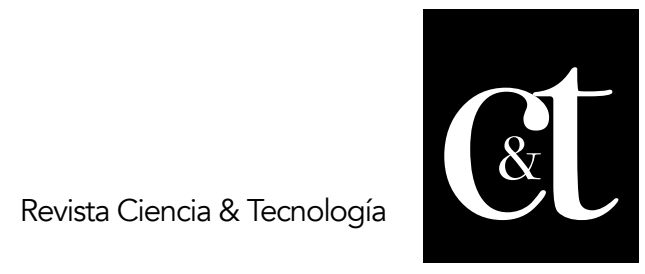

No. 30, 30 de abril de 2021

ISSN impreso: 1390 - 6321

ISSN online: 2661 - 6734

Se consideró como caso de estudio la población de 20 docentes pertenecientes a la comunidad de profesores de la Universidad Laica "Eloy Alfaro" de Manabí (ULEAM), ubicada en Manta, Ecuador, específicamente de la carrera Ingeniería de Sistemas, Facultad de Ciencias Informáticas, la condición de la selección de estos individuos fue que estuvieran utilizando plataformas virtuales para e-learning. Por ser una población pequeña o de pocos individuos, se consideró en su totalidad para el estudio.

La recolección de la información, se realizó mediante la aplicación de la técnica de la encuesta a los docentes antes mencionados, como instrumento se aplicó un cuestionario (ver anexo) diseñado atendiendo a seis dimensiones enfocadas a las herramientas, las cuales son: 1 . Orientadas al aprendizaje; 2 . Orientadas a la productividad; 3. Para la implicación de los estudiantes; 4. Destinadas a la publicación de cursos y contenidos; 5 . Para el diseño de planes de estudio y 6 . Para la gestión del conocimiento.

La misma quedó estructurada en 45 proposiciones cerradas con cinco alternativas de respuesta cada una (5: Muy frecuentemente; 4: Frecuentemente; 3: Algunas veces; 2: Casi nunca y1: Nunca) y una pregunta abierta. Fue validada por expertos en el área y determinada su confiabilidad, obteniendo el índice de fiabilidad mediante al alfa de Cronbach, el cual fue de 0.932, lo cual permite concluir que el índice de fiabilidad del instrumento es muy alta según Palella y Martins (2012).

\section{Resultados y discusión}

La aplicación del cuestionario de recolección de información permitió determinar las preferencias de docentes universitarios sobre herramientas para plataformas de e-learning, a continuación se presentan los resultados organizados en tablas relacionadas a las dimensiones de la investigación, de tal forma que la tabla 1 corresponde a la dimensión 1: herramientas orientadas al aprendizaje; para la dimensión 2: herramientas orientadas a la productividad se presenta la tabla 2; la tabla 3, asociada a la dimensión 3: herramientas de implicación de estudiantes; para la dimensión 4: herramientas destinadas a la publicación de cursos y contenidos, se muestra la tabla 4; con relación a la dimensión 5: herramientas para el diseño de planes de estudio, se presenta la tabla 5 y la tabla 6 corresponde a la dimensión 6: herramientas de gestión del conocimiento.

Como primera información se les preguntó sobre la plataforma virtual utilizada en su hacer educativo. Al respecto, los encuestados mencionaron varias, entre ellas: Zoom, Teams, Moodle, Ofice 365, Meet, Aula virtual, Schoology, WhatsApp, Scream y Youtube, siendo las preferidas Zoom, Teams con un porcentaje de $80 \%$ y $75 \%$ respectivamente. Este resultado coincide con Sánchez (2020), quien opina que existen muchas herramientas, pero probablemente la más utilizada es la plataforma de Zoom, ideal para videoconferencias, la cual ha adquirido una importancia inusitada por su facilidad de uso, por su compatibilidad con todos los sistemas operativos y porque se puede utilizar en todo tipo de dispositivos digitales. Además se opone al resultado obtenido por Arroyo et al. (2018), quienes opinaron que Moodle era la plataforma preferida para la fecha. A continuación se presentan las tablas de frecuencias y porcentajes de cada una de las dimensiones estudiadas.

\section{5}

García, López, Mora, Pinargote, García. Preferencias de docentes universitarios sobre herramientas para plataformas de e-learning 


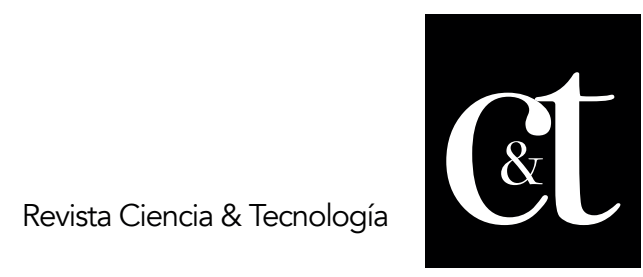

No. 30, 30 de abril de 2021

ISSN impreso: 1390 - 6321

ISSN online: 2661 - 6734

Tabla 1. Frecuencias y porcentajes de respuestas a proposiciones de la dimensión 1

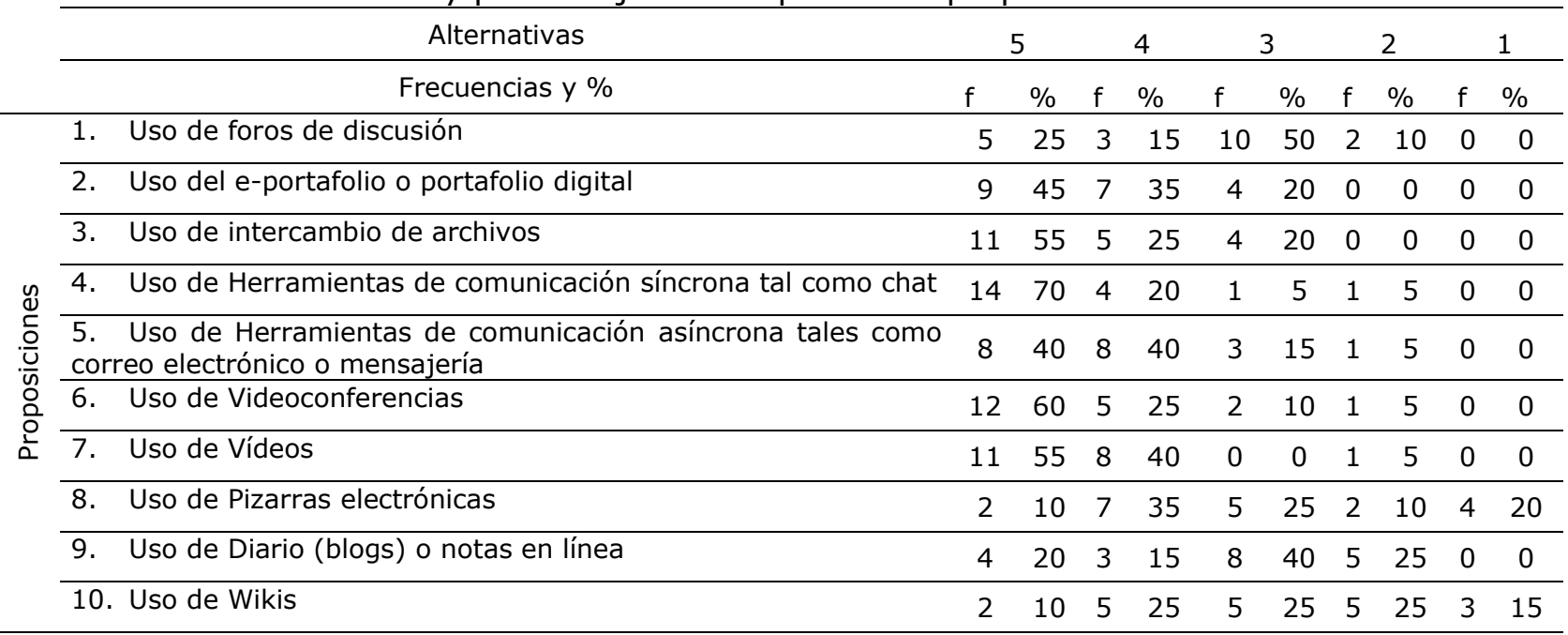

Fuente: elaboración propia

Los resultados de la tabla 1 evidencian la preferencia por el uso de herramientas de comunicación síncrona tal como el chat con un $70 \%$, seguido por el uso de videoconferencias con un $60 \%$, el intercambio de archivos y el uso de videos con un $55 \%$, como herramientas orientadas al aprendizaje, las cuales permiten el intercambio de información con mayor precisión y rapidez. Este resultado contradice los presentados por Quimiz (2018), quien encontró que el chat prácticamente no se utilizan, incluso ni por los docentes de la carrera de sistemas. Con relación al uso de videoconferencias concuerda con su preferencia por el uso de zoom. Seguidamente se muestra la tabla 2, con los resultados de las respuestas de los docentes a la encuesta administrada, expresados en frecuencias y porcentajes de la dimensión 2: herramientas orientadas a la productividad.

Tabla 2. Frecuencias y porcentajes de respuestas a proposiciones de la dimensión 2

\begin{tabular}{|c|c|c|c|c|c|c|c|c|c|c|c|}
\hline & Alternativas & \multicolumn{3}{|c|}{5} & \multicolumn{2}{|c|}{4} & 3 & \multicolumn{2}{|r|}{2} & \multicolumn{2}{|c|}{1} \\
\hline & Frecuencias y \% & $f$ & $\%$ & $f$ & $\%$ & $\mathrm{~F}$ & $\%$ & $f$ & $\%$ & $f$ & $\%$ \\
\hline \multirow{10}{*}{ 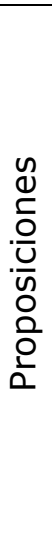 } & 11. Uso de Bookmarks & 1 & 5 & 4 & 20 & 2 & 10 & 5 & 25 & 8 & 40 \\
\hline & 12. Uso de calendario y revisión del progreso & 6 & 30 & 5 & 25 & 5 & 25 & 2 & 10 & 2 & 10 \\
\hline & 13. Uso de tutoriales, manuales de usuario, ayuda en línea & 10 & 50 & 5 & 25 & 5 & 25 & 0 & 0 & 0 & 0 \\
\hline & 14. Uso del buscador de cursos mediante un patrón de búsqueda & 5 & 25 & 9 & 45 & 4 & 20 & 0 & 0 & 2 & 10 \\
\hline & 15. Uso de mecanismos de sincronización y trabajo fuera de línea & 5 & 25 & 5 & 25 & 8 & 40 & 2 & 10 & 0 & 0 \\
\hline & 16. Uso de control de publicación, páginas caducadas y enlaces rotos & 2 & 10 & 4 & 20 & 5 & 25 & 3 & 15 & 4 & 20 \\
\hline & 17. Uso de noticias del lugar & 3 & 15 & 7 & 35 & 5 & 25 & 5 & 25 & 0 & 0 \\
\hline & 18. Uso de visos de actualización de páginas & 2 & 10 & 4 & 20 & 5 & 25 & 8 & 40 & 1 & 5 \\
\hline & 19. Uso de mensajes a foros y envío automático & 4 & 20 & 4 & 20 & 5 & 25 & 6 & 30 & 1 & 5 \\
\hline & 20. Uso de soporte a la sindicación de contenidos & 1 & 5 & 7 & 35 & 5 & 25 & 6 & 30 & 1 & 5 \\
\hline
\end{tabular}

Fuente: elaboración propia 


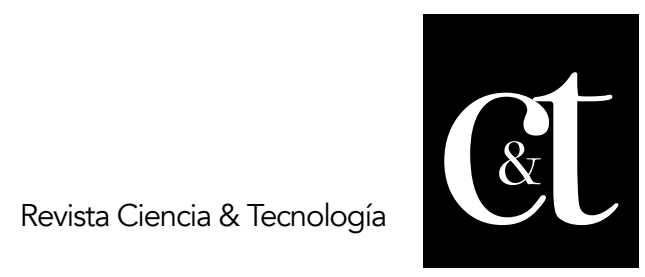

No. 30,30 de abril de 2021

ISSN impreso: 1390 - 6321

ISSN online: 2661 - 6734

Como herramientas orientadas a la productividad (dimensión 2), en la tabla 2 se muestra la preferencia por el uso de tutoriales, manuales de usuario, ayuda en línea con un $50 \%$. Es decir, estos docentes prefieren herramientas de orientación y ayuda a los participantes en el uso del sistema de aprendizaje (Boneu, 2007). A continuación se presenta la tabla 3 , la cual resume las frecuencias y porcentajes de las respuestas a las proposiciones correspondientes a la dimensión 3: herramientas de implicación de estudiantes.

Tabla 3. Frecuencias y porcentajes de respuestas a proposiciones de la dimensión 3

\begin{tabular}{|c|c|c|c|c|c|c|c|c|c|c|c|}
\hline & \multirow{2}{*}{$\begin{array}{c}\text { Alternativas } \\
\text { Frecuencias y \% }\end{array}$} & \multicolumn{2}{|c|}{5} & \multicolumn{2}{|c|}{4} & \multicolumn{2}{|c|}{3} & \multicolumn{2}{|c|}{2} & \multicolumn{2}{|c|}{1} \\
\hline & & $f$ & $\%$ & $f$ & $\%$ & $f$ & $\%$ & $f$ & $\%$ & $f$ & $\%$ \\
\hline \multirow{8}{*}{ 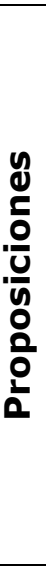 } & 21. Uso de grupos de trabajo & 7 & 35 & 7 & 35 & 3 & 15 & 3 & 15 & 0 & 0 \\
\hline & 22. Uso de las autovaloraciones & 4 & 20 & 10 & 50 & 5 & 25 & 0 & 0 & 1 & 5 \\
\hline & $\begin{array}{l}\text { 23. Uso del rincón del estudiante, o espacios para hacer grupos de } \\
\text { estudio, clubes o equipos de trabajo colaborativos }\end{array}$ & 4 & 20 & 3 & 15 & 5 & 25 & 6 & 30 & 2 & 10 \\
\hline & $\begin{array}{l}\text { 24. Uso del perfil del estudiante, o espacios para mostrar el trabajo } \\
\text { en un curso, anunciarse, mostrar su fotografía, preferencias, } \\
\text { temas de interés o información personal. }\end{array}$ & 6 & 30 & 7 & 35 & 3 & 15 & 2 & 10 & 2 & 10 \\
\hline & $\begin{array}{l}\text { 25. Uso de la autenticación de usuarios, mediante el nombre de } \\
\text { usuario y contraseña }\end{array}$ & 15 & 75 & 3 & 15 & 2 & 10 & 0 & 0 & 0 & 0 \\
\hline & 26. Uso de la asignación de privilegios en función del rol del usuario & 10 & 50 & 3 & 15 & 3 & 15 & 2 & 10 & 2 & 10 \\
\hline & 27. Uso del registro de estudiantes & 14 & 70 & 5 & 25 & 1 & 5 & 0 & 0 & 0 & 0 \\
\hline & $\begin{array}{l}\text { 28. Uso de la auditoría, o herramientas que permiten consultar } \\
\text { todas las acciones realizadas por los participantes de la } \\
\text { plataforma, así como obtener estadísticas sobre su utilización }\end{array}$ & 7 & 35 & 4 & 20 & 5 & 25 & 2 & 10 & 2 & 10 \\
\hline
\end{tabular}

Los resultados de la tabla 3 evidencian la preferencia de las herramientas de implicación de estudiantes, en esta dimensión, los docentes encuestados se inclinaron en su mayoría por el uso de la autenticación de usuarios, mediante el nombre de usuario y contraseña con un $75 \%$, seguido por el uso del registro de estudiantes con un $70 \%$, y el uso de la asignación de privilegios en función del rol del usuario con un $50 \%$. Con relación al uso de grupos de trabajo, las opiniones de los docentes encuestados quedaron divididas entre muy frecuentemente (35\%) y frecuentemente (35\%); es importante resaltar que esta herramienta favorece la aplicación de estrategias para lograr el aprendizaje colaborativo, según Boneu (2007), esta herramienta permite organizar una clase en grupos, donde el profesor asigna tareas o actividades pertinentes. A continuación se muestra la tabla 4, con las frecuencias y porcentajes de las proposiciones correspondientes a la dimensión 4: herramientas destinadas a la publicación de cursos y contenidos.

García, López, Mora, Pinargote, García. Preferencias de docentes universitarios sobre herramientas para plataformas de e-learning 


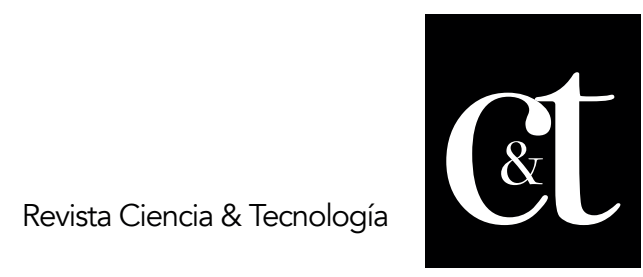

No. 30,30 de abril de 2021

ISSN impreso: 1390 - 6321

ISSN online: 2661 - 6734

Tabla 4. Frecuencias y porcentajes de respuestas a proposiciones de la dimensión 4

\begin{tabular}{|c|c|c|c|c|c|c|c|c|c|c|c|}
\hline & Alternativas & & 5 & & 4 & & 3 & & 2 & & 1 \\
\hline & Frecuencias y \% & f & $\%$ & $f$ & $\%$ & $\mathrm{f}$ & $\%$ & $f$ & $\%$ & $f$ & $\%$ \\
\hline \multirow{5}{*}{$\begin{array}{l}\text { D } \\
\frac{0}{0} \\
\frac{0}{0} \\
\frac{0}{0} \\
\frac{0}{2}\end{array}$} & 29. Uso de los test y resultados automatizados & 9 & 45 & 4 & 20 & 4 & 20 & 3 & 15 & 0 & 0 \\
\hline & 30. Uso de la administración del curso & 9 & 45 & 7 & 35 & 2 & 10 & 2 & 10 & 0 & 0 \\
\hline & 31. Uso del apoyo al creador de cursos & 5 & 25 & 9 & 45 & 3 & 15 & 3 & 15 & 0 & 0 \\
\hline & 32. Uso de las herramientas de calificación en línea & 12 & 60 & 4 & 20 & 3 & 15 & 1 & 5 & 0 & 0 \\
\hline & 33. Uso del seguimiento del estudiante & 14 & 70 & 2 & 10 & 4 & 20 & 0 & 0 & 0 & 0 \\
\hline
\end{tabular}

Fuente: elaboración propia

Como herramientas destinadas a la publicación de cursos y contenidos (dimensión 4 ), en la tabla 4 se muestra la preferencia por el uso del seguimiento del estudiante con un $70 \%$ y el uso de las herramientas de calificación en línea con un $60 \%$. Esto es, según palabras de Boneu (2007), estos docentes prefieren herramientas que proporcionan un análisis adicional sobre el uso que se hace de los materiales del curso por parte de los estudiantes y de las calificaciones que obtienen a utilizar test y resultados automatizados como evaluaciones. Seguidamente se presenta la tabla 5 , con las frecuencias y porcentajes de las proposiciones correspondientes a la dimensión 5: herramientas para el diseño de planes de estudio.

Tabla 5. Frecuencias y porcentajes de respuestas a proposiciones de la dimensión 5

\begin{tabular}{|c|c|c|c|c|c|c|c|c|c|c|c|}
\hline & Alternativas & \multicolumn{2}{|c|}{5} & \multicolumn{2}{|c|}{4} & \multicolumn{2}{|r|}{3} & \multicolumn{2}{|r|}{2} & \multicolumn{2}{|c|}{1} \\
\hline & Frecuencias y \% & $f$ & $\%$ & $f$ & $\%$ & $f$ & $\%$ & $f$ & $\%$ & $f$ & $\%$ \\
\hline \multirow{7}{*}{ 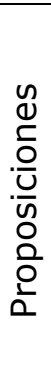 } & 34. Uso de la conformidad con la accesibilidad & 6 & 30 & 7 & 35 & 5 & 25 & 2 & 10 & 0 & 0 \\
\hline & 35. Uso de la reutilización y compartición de contenidos & 10 & 50 & 6 & 30 & 2 & 10 & 2 & 10 & 0 & 0 \\
\hline & $\begin{array}{l}\text { 36. Uso de las plantillas de curso, útiles para crear la estructura de } \\
\text { un curso en línea }\end{array}$ & 7 & 35 & 4 & 20 & 7 & 35 & 2 & 10 & 0 & 0 \\
\hline & 37. Uso de la administración del currículum & 6 & 30 & 8 & 40 & 3 & 15 & 2 & 10 & 1 & 5 \\
\hline & 38. Uso de la personalización del entorno & 9 & 45 & 7 & 35 & 1 & 5 & 3 & 15 & 0 & 0 \\
\hline & 39. Uso de las herramientas para el diseño de la educación & 8 & 40 & 6 & 30 & 5 & 25 & 0 & 0 & 1 & 5 \\
\hline & 40. Uso de la & 6 & 30 & 8 & 40 & 5 & 25 & 0 & 0 & 1 & 5 \\
\hline
\end{tabular}

Fuente: elaboración propia

Como herramientas para el diseño de planes de estudio (dimensión 5), en la tabla 5 se muestra la preferencia por el uso de la reutilización y compartición de contenidos con un 50\%. Boneu (2007), opina que estos docentes prefieren que los contenidos creados por un curso puedan ser compartidos con otro profesor, en otro curso y otro centro. A continuación se evidencia la tabla 6, con las frecuencias y porcentajes de las proposiciones correspondientes a la dimensión 6: herramientas de gestión del conocimiento. 


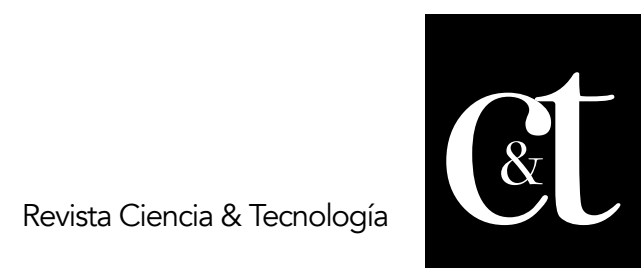

No. 30,30 de abril de 2021

ISSN impreso: 1390 - 6321

ISSN online: 2661 - 6734

Tabla 6. Frecuencias y porcentajes de respuestas a proposiciones de la dimensión 6

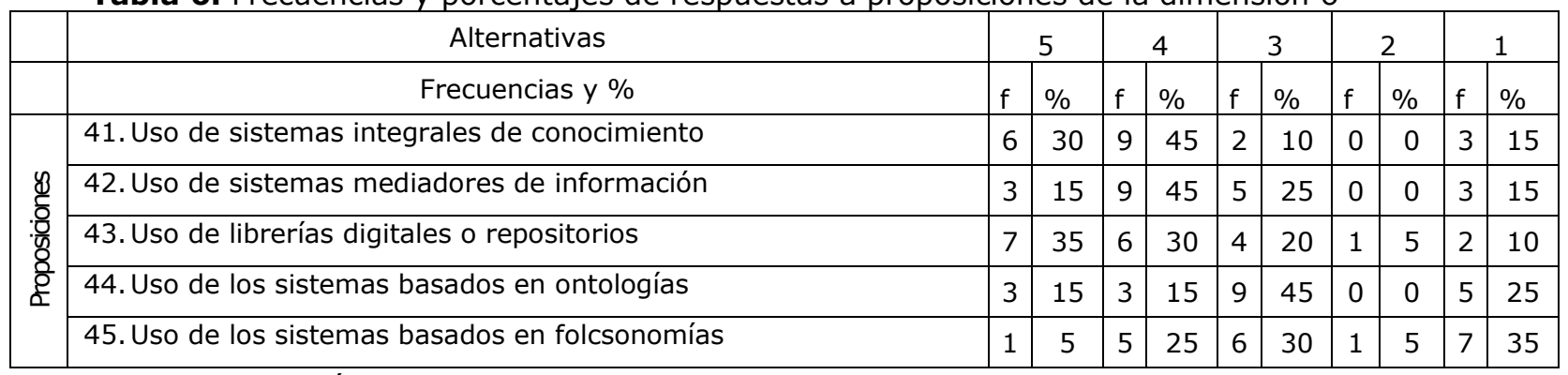

Fuente: elaboración propia

Como herramientas de gestión del conocimiento (dimensión 6), en la tabla 6, se unen las alternativas de respuesta 5 y 4 ; esto es, las respuestas muy frecuentemente y frecuentemente, y se evidencia la preferencia por el uso de sistemas integrales de conocimiento con un $75 \%$, el uso de librerías digitales o repositorios $(65 \%)$ y el uso de sistemas mediadores de información con un $60 \%$. Esto es, según Boneu (2007), afirma que las preferencias de estos docentes están vinculadas a sistemas cuyas herramientas normalmente representan la estructura del conocimiento de forma jerárquica, en lo que se conoce como árbol de conocimiento; o mediante la representación del conocimiento como redes jerárquicas de nodos conectados entre sí por relaciones. Además gustan de herramientas que permiten recolectar información desde fuentes distribuidas y le permiten construir al usuario su propia biblioteca digital.

Como resumen de los resultados de todas las dimensiones y tomando el máximo valor del porcentaje de respuestas, se presenta la tabla 7.

Tabla 7. Máximo valor del porcentaje de respuestas en todas las dimensiones

\begin{tabular}{lllc}
\hline \multicolumn{1}{c}{ Dimensiones } & \multicolumn{1}{c}{ Proposiciones } & $\begin{array}{c}\text { Porcentaje de } \\
\text { respuestas }\end{array}$ \\
\hline $\begin{array}{l}\text { Dimensión 1: Herramientas orientadas al } \\
\text { aprendizaje }\end{array}$ & $\begin{array}{l}\text { Uso de Herramientas de comunicación síncrona } \\
\text { tal como chat }\end{array}$ & $70 \%$ \\
\hline $\begin{array}{l}\text { Dimensión 2: Herramientas orientadas a la } \\
\text { productividad }\end{array}$ & $\begin{array}{l}\text { Uso de tutoriales, manuales de usuario, ayuda } \\
\text { en línea }\end{array}$ & $50 \%$ \\
\hline $\begin{array}{l}\text { Dimensión 3: Herramientas de implicación de } \\
\text { estudiantes }\end{array}$ & $\begin{array}{l}\text { Uso de la autenticación de usuarios, mediante } \\
\text { el nombre de usuario y contraseña }\end{array}$ & $75 \%$ \\
\hline $\begin{array}{l}\text { Dimensión 4: Herramientas destinadas a la } \\
\text { publicación de cursos y contenidos }\end{array}$ & Uso del seguimiento del estudiante & $70 \%$ \\
\hline $\begin{array}{l}\text { Dimensión 5: Herramientas para el diseño de } \\
\text { planes de estudio }\end{array}$ & $\begin{array}{l}\text { Uso de la reutilización y compartición de } \\
\text { contenidos }\end{array}$ & $50 \%$ \\
\hline $\begin{array}{l}\text { Dimensión 6: Herramientas de gestión del } \\
\text { conocimiento }\end{array}$ & Uso de sistemas integrales de conocimiento & $75 \%$ \\
\hline
\end{tabular}

Fuente: elaboración propia

\section{Conclusiones}

Con la educación cambiando y adaptándose a la modalidad virtual o e-learning, los docentes universitarios se han visto en la necesidad de usar plataformas que mejor se adapten a sus necesidades y preferencias de acuerdo a las características de las asignaturas que administran. Estas plataformas han sido consideradas por los docentes como herramientas tecnológicas con fuertes potencialidades para su incorporación a la enseñanza.

\section{9}

García, López, Mora, Pinargote, García. Preferencias de docentes universitarios sobre herramientas para plataformas de e-learning 


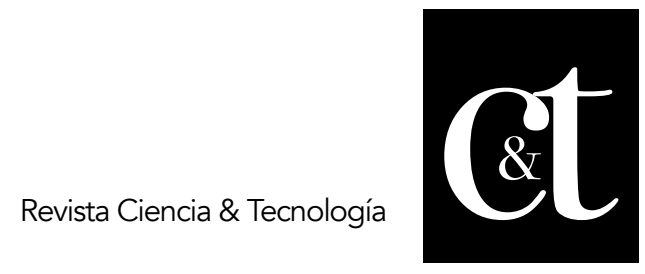

No. 30, 30 de abril de 2021

ISSN impreso: 1390 - 6321

ISSN online: 2661 - 6734

Esta investigación tuvo como objetivo determinar las preferencias de docentes universitarios sobre herramientas para plataformas de e-learning, consiguiendo que las preferencias de acuerdo a las dimensiones estudiadas están en sintonía con las plataformas usadas: Zoom, Teams. Los motivos de la no utilización, o uso con poca frecuencia de las herramientas pueden ser diversos, tal como la complejidad de la plataforma, la falta de conocimiento sobre su funcionamiento y potencialidades, el considerar que la herramienta no se adapta para el uso educativo, o por las actitudes que los docentes tengan hacia la tecnología a usar. Ante lo cual se recomienda continuar indagando sobre estos aspectos mencionados, así como investigar también las preferencias de los estudiantes.

\section{Referencias}

Arroyo, Z.; Fernández, S.; Barreto, L. \& Paz, L. (2018). Entornos virtuales de aprendizaje en comunidades de práctica de docentes universitarios del Ecuador. Revista Ensayos Pedagógicos, XIII(2), 185-200.

Boneu, J. (2007). Plataformas abiertas de e-learning para el soporte de contenidos educativos abiertos. RUSC Universities and Knowledge Society Journal, 4(1), 36-47.

Chávez, V. \& Verdezoto, R. (2018). Importancia de las herramientas y entornos de aprendizaje dentro de la plataforma e-learning en las universidades de Ecuador. EDUTEC Revista Electrónica de Tecnología Educativa, 65, pp. 6892. DOI: http://dx.doi.org/10.21556/edutec.2018.65.1067.

Consejo de Educación Superior. (2019). Reglamento del Régimen Académico del Ecuador. RPC-SO-08-No.111-2019, Quito, Ecuador.

Coogle. Plataformas abiertas de e learning gratuitas para e learning. Obtenido de https://coggle.it/diagram/WttKzIIXs6kD4kv7/t/plataformas-abiertas-de-elearning-gratuitas-para-e-learning

Del Prete, A. \& Cabero, J. (2019). Las plataformas de formación virtual: algunas variables que determinan su utilización. Revista Apertura, 11(2), pp. 20-35. DOI: http://dx.doi.org/10.18381/Ap.v11n2.1521.

Gallardo, A. (2020). Guía de trabajo. TFM Alejandro Gallardo doc.x

Gros, B. (2018). La evolución del e-learning: del aula virtual a la red. RIED Revista Iberoamericana de Educación a Distancia, 21(2), 69-82. DOI: http://dx.doi.org/10.5944/ried.21.2.20577.

Guevara, E., Pardo, M. \& Izquierdo, J. (2017). Reflexión acerca de la virtualización académica universitaria en la Universidad Estatal de Bolívar en Ecuador. Revista Didasc@lia, 8(7), 1-7. https://runachayecuador.com/refcale/index.php/didascalia/article/view/1864

Hernández, R.; Fernández, C. \& Baptista, P. (2014). Metodología de la investigación. Sexta edición, México: McGraw Hill/Interamericana Editores S.A. de C.V.

Ilgaz, H., \& Gulbahar, Y. (2017). Why do learners choose online learning: the learners' voices. International Conference On E-Learning, 130-136. http://search.ebscohost.com/login. aspx?direct=true\&db=asn\&AN=12702285 9\&lang=es\&site $=$ ehost-live.

Martínez, A., Mauri, T., Colomina, R., Agirre, N., Marc, C., Bilbatua ,M., Onrubia, J. y Pablo, G. (2017). Factores que favorecen la presencia docente en entornos virtuales de aprendizaje. Tendencias Pedagógicas, 3, 43-58. https://revistas.uam.es/tendenciaspedagogicas/ article/view/7083.

Palella, S. \& Martins, F. (2012). Metodología de la investigación cuantitativa. Caracas, Venezuela: Fondo Editorial de la Universidad Pedagógica Experimental Libertador. 


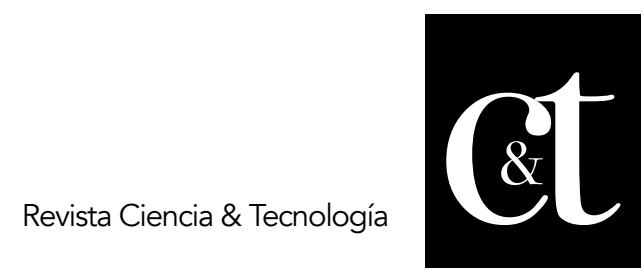

No. 30, 30 de abril de 2021

ISSN impreso: 1390 - 6321

ISSN online: 2661 - 6734

Quimiz, J. (2018). Entorno virtual de aprendizaje en la Universidad Regional Autónoma de los Andes Extensión Santo Domingo [tesis de maestría, Universidad Regional Autónoma de los Andes "UNIANDES"], Santo Domingo, Ecuador.

Rea, M. \& Medina, J. (2016). La educación a distancia como mecanismo de inclusión social en las universidades del Ecuador. Alternativas, 17(2), 5-10. https://editorial.ucsg.edu.ec/ojs-alternativas/index.php/alternativasucsg/article/view/113.

Sánchez, M. (2020). Involucrar a los estudiantes al usar Zoom. Revista Mexicana de Bachillerato a Distancia, 24(12), 1-3. http://revistas.unam.mx/index.php/rmbd/article/view/76818/67843.

García, López, Mora, Pinargote, García. Preferencias de docentes universitarios sobre herramientas para plataformas de e-learning 